\title{
A Study of Performance Evaluation of Convolution Neural Network for Diabetic Retinopathy
}

\author{
Kusuma R C ${ }^{1}$, Shilpa Ankalaki², Dr Jharna Majumdar ${ }^{3}$ \\ ${ }^{1} \mathrm{M}$ Tech Scholar, NMIT, India, kusumakusu080@gmail.com \\ ${ }^{2}$ Assistant Professor, NMIT, India, shilpa.a@nmit.ac.in \\ ${ }^{3}$ Dean R\&D, Professor, NMIT, India, jharna.majumdar@gmail.com
}

\begin{abstract}
Diabetic retinopathy is a retinal illness, caused by high blood sugar level, which will lead to damage in retinal blood vessels. It is difficult to detect the disease in early stages, as it will not show any symptoms at the early stage. DR is the major reason for the blindness in Diabetic patients. Clinicians recommends that the patient's needs to be diagnosed at least two times per year in order to identify signs of disease caused by diabetic retinopathy. At present diagnosis for the detection of the diabetic retinopathy mainly depends on ophthalmologist who examines the retinal image and decides the patient's condition that they have diabetic retinopathy or not. Computer-aided verification of fundus images is necessary because it permits for fast processing and bunch images can be analyzed in one shot at a time. Consequently, to attain fast, reliable diagnosis and totreat the patient at the early stages application of deep CNNs approaches are used. Automatic diabetic retinopathy detection examines DR fundus images into four categories called No DR, Mild DR, Moderate DR, severe DR and proliferative DR. Diabetic Retinopathy can be found in earlier stage, will be treated immediately and in early detection Deep Convolutional Neural Networks are playing very important role. In the proposed Methodology ResNet-50, ResNet-101, VGG-16 and Inception-V3 neural networks are trained on APTOS dataset form Kaggel. To analyzing the performance, all models in the study trained on same dataset. Fine-tuning achieved to increase the performance of proposed methodology.
\end{abstract}

Key words : Diabetic Retinopathy, Diagnosed, Ophthalmologist, Computer Vision, Computer-Aided Diagnosis, CNN, ResNet-50, ResNet-101, VGG-16 and Inception-v3.

\section{INTRODUCTION}

Diabetic retinopathy is one of retinal complication results when blood sugar level is very high, it is origin for the other problems in diabetic patients such as coronary infraction, stroke, diabetic neuropathy and diabetic nephropathy. Diabetes mellitus or hypoglycemia is injury in retinal capillaries and envisioned in retina of the eye [1]. Retina is a thin layer, which is sensitive to light, present in backside of eyeball. Diabetic retinopathy causes vision disruption that may result to permanent myopia or astigmatism (blindness). The effect of manual inspection of diabetic retinopathy is mainly dependent on the ophthalmologist's experience and it is time consuming. Disguise about disease frequently exists sometimes due to the less experience of doctors. From the past few years, Deep Convolutional Neural Networks made very good achievements in computer vision and image classification methodologies compared to the previous used methodologies.

Accordingly, a successful diabetic retinopathy screening is necessary to provide the treatment from early stages of the disease. As per survey in the year of 2014 there were 422 million individuals living with hypoglycemia. In the year, 2010 almost one third of humans were having high blood sugar level, which shows signs of Diabetic Retinopathy (DR) [2]. Studies on DR says, patients will be triple by the year 2050, and most of them will be around 65 years or more [3]. There are mainly five stages of Diabetic Retinopathy. First stage is 'No Diabetic Retinopathy' where there will not be any damages caused by Diabetes mellitus to the retina of the eye. The 'mild DR' is second stage of Diabetic Retinopathy this is when micro aneurysms start to appear, one of the primary symptom to identify the diabetic retinopathy in the early phase form fundus images.. These images will labelled under 'mild DR' [4]. Appearance of blot hemorrhages and dots, multiple micro aneurysms and cotton wool spots, hard exudates in fundus are cause for 'Moderate DR', which is third stage.

Stage four of DR is distinguished by intraretinal microvascular irregularity along with more damages in retina, belongs to the 'severe DR'. In final stage, retinal detachment may start appearing along with the growth of new retinal blood vessels and blood starts leaking into the vitreous humor of eye. Most commonly used diagnose techniques from ophthalmologist are fluorescein angiography, fundus photography and optical coherence tomography [5]. In this study, Kaggel diabetic retinopathy images from APTOS are used as Dataset. CNN is a class of DNN, it will take image as input, gives significance to many entity in the image and able to find difference from one object and another object. 
Compared to other classification algorithms $\mathrm{CNN}$ requires very less pre-processing. Learning the filters or characteristics on its own ability of Convolutional Neural Network, whereas ancient networks filters are hand-engineered. In human brain, there is a limited region of field of the vision, which is called as Receptive field, refers to single neurons response to stimuli [6]. The connectivity pattern of neurons in Human Brain is analogous to the Architecture of Convolutional Neural Network and Visual Cortex is one inspired by the organization. Collections of Visual fields imbricate to shield the entire complete region. Transfer learning in Deep learning and Machine Learning is a technique, where a neural network model that is first developed and trained on some other dataset for a particular problem, then used for similar kind of problems [7]. The pre-trained model can be trained on different dataset for solving different problems instead of training from scratch. Transfer learning is most popular approach for NLP and computer vision tasks in deep learning.

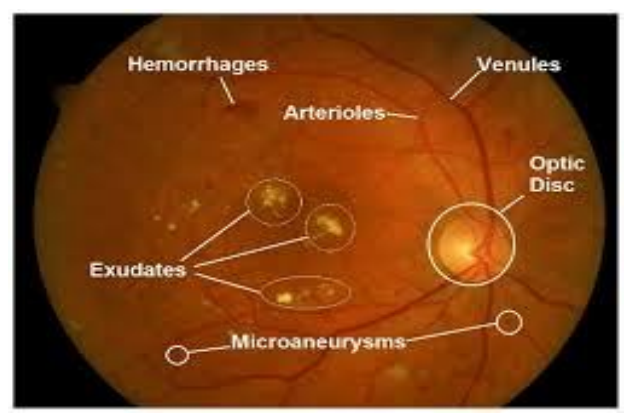

Figure 1: Features to Identity Diabetic Retinopathy.

\section{LITERATURE SURVEY}

The Deep Convolutional Neural Networks recently achieved significant victory in field of computer-vision. CNN architectures, namely ResNet, GoogLeNet and VGGNet, achieved the best validation accuracy in ILSVRC [7]. In addition, DCNN's grades DR in 5 classes, No DR or normal, mild, Moderate, Severe DR and Prolific DR using DCNN. These networks will pre-segment given input and predicts normal or abnormal condition of the image based on the features like hemorrhages, micro aneurysms and hard exudates, growth of new retinal blood vessels and leakage of blood in blood vessels. VGG16 network on Kaggel DR Fundus image dataset [8], achieved accuracy of 0.75 on 3662 test images. Deep CNNs used mainly for classification of the images in to respective classes based on the condition of the image. Inception-v3 is 48 layers deep Convolutional Neural Network. The Inception-V3 Network framework incorporated with two max-pooling layers, five convolutional (conv) layers, one average-pooling layer, eleven inception modules followed by a fully connected layer, which produces an image-wise array. As the width and depth of the network increases the computation activity lessen, Inception v3 clusters the similar sparse nodes into dense structure. To gain knowledge from learning to complete one task is leveraged to complete another task of same structure is called Transfer learning [9]. If a model is built to classify the truck, it follows that it can identify parts of the model to classify the cars. In some cases if the domains are not same, still have good results with Transfer learning [10]. Comparatively training a model from scratch will give good performance but it will be cost effective as well as takes time to train a network from scratch. Transfer learning also leads to good performance with the learning and no need to train a network from scratch. Accurate binary classifier to differentiate the fundus image based on injury is main objective [11]. Aim is to classify fundus images into five classes based on the injuries and symptoms, to identify the fundus image that belongs to DR or non-DR. In the proposed methodology different types of deep convolutional neural networks as our learning mode, which are current state of art for images classification [12]. Figure.2. Depicts the classification fundus images based on the amount of injury. Images are mainly classified into 5 major classes. Image A belongs to the normal class, B belongs to the class Mild DR, C belongs to the class moderate, D is the fundus image of the patient belongs to the class severe and $\mathrm{E}$ represents the image which belongs to the class proliferative DR.

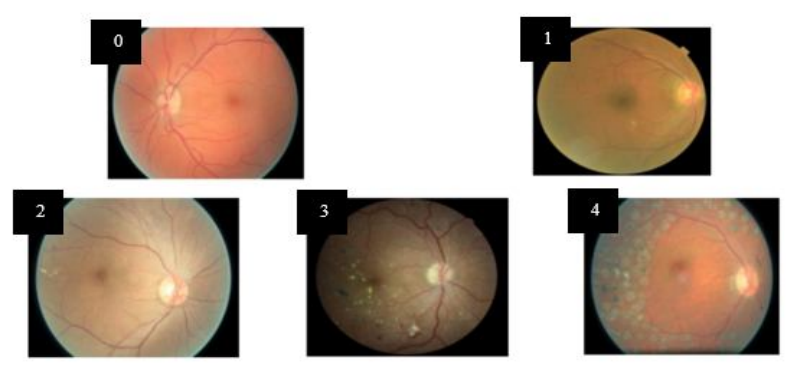

Figure 2: Retinal images: [0] Normal [1] Mild [2] Moderate [2] Severe [4] Proliferative DR.

\section{DATA SET}

The Dataset is from APTOS Diabetic Retinopathy Detection Competition from Kaggel website. Dataset contains retinal fundus images taken in variety of imaging stages, and the dataset comprise high-resolution retinal fundus images of huge set. The clinical experts has evaluated the amount of disease in images with scale of 0 to 4 is as follows:
[0] - No DR
[1] - Mild
[2] - Moderate
[3] - Severe
[4] - Proliferative DR 
Ophthalmologists identify DR images with the injuries and wounds corresponding to vascular injuries caused by the Diabetic Retinopathy. As this approach is successful, but resource required for this approach are very high. Because of lacking in the expertise and equipment, rate of diabetes in population is more. Early and automatic detection of diabetic retinopathy is more required. The fundus images captured from different kind of camera models [13]. Type of the camera decides the visual appearance of the fundus images. Below is the Link for the already available Kaggel Diabetic Retinopathy 2015 and 2019 Datasets: https://www.kaggle.com/benjaminwarner/resized-2015-2019 -blindness-detection-images

\section{DATA PRE-PROCESSING}

Loading of input diabetic retinopathy fundus images and resizing the images to $(224,224)$. Added the channels (224, $224,3)$ for the RGB and $(224,224,1)$ for gray images. Data format can be passed as an argument, to put the channels either at the first axis (or) at the last axis. The Dimension expansion is used to add the number of images for example $(1,224,224,3)$. Subtracted mean RGB channels of the ImageNet dataset. To create batch of images needs a further dimension of (samples, size1, size2 channels). The preprocessing is used to make input images sufficient into format that model requires. Some of the models uses images with the range 0 to 1 and some with range -1 and 1 and some with 'caffe' style. In the proposed methodology, images are loaded with the help of keras functions (So we have guarantee that the images you load are compatible with the model after preprocessing). In this study, pre-trained networks on ImageNet dataset are used. The submitting author is responsible for obtaining agreement of all coauthors and any consent required from sponsors before submitting a paper. It is the obligation of the authors to cite relevant prior work.

\section{PROPOSED METHODOLOGY}

The proposed methadology is based on Transfer learning with deep learning, so the deep neural network models that are trained on Imagenet Data is used for some of their advantages like they are simple to incorporate, models will achieve very good performance fastly, there is no much labeled data required and there are flexibe use cases from transfer learning, prediction and feature extraction.To optimize the performance of the proposed model, tarining accuarcy and prediction capacity of the trained models in this study, fine tuning is achieved by adding fully-connected, dropout and dense layers to existing Neural network models. In the process fo fine tuning one global spatial average pooling layer, three fully connected and three dropout layers, with 3 dense layers and 3 dropout layers are added. Relu activation function and Leaky Relu actiavtion function with alpha value 0.001 and an output layer with a softmax classifier is added to classify theimages into 4 classes. To prevent the weights in a given layer from being updated during training freezing is required. It is key to freeze the convolution base before compiling and training a neural network.

The Figure. 3 depicts the flow chart for the methodology used in the study, firstly the Retinal fundus images are given as input to the neural network. Next step is preprocessing where the images are resized as colour image channel $(224,224,3)$. Then the Deep Neural networks such as ResNet-50, ResNet-101,VGG-16 and Inception-V3 are trained for the given input, transer learning is achieved, by downloading the weights of the pre-trained networks then use the newly trained network for the DR detection purpose. Finally testing the trained model and then the trained model can be use for the prediction of DR. Finetuning is achieved to get better results from the neural network models. Added average pooling layer, fully-connected layer and dropout layers. Good results form VGG-16 with finetuning.

In the proposed methodology, different types of pre-trained Deep Convolutional Neural Networks were trained on 3662 training images, tested on 1928 test images from Kaggel diabetic retinopathy fundus images. ResNet-50 and Resnet-101 were trained on 35126 Kaggel 2015 resized images because the dataset was very much imbalanced. More images that belong to No DR, Mild, Moderate and for Severe and Proliferative DR images are less in number compared to first three classes, so the prediction was poor.

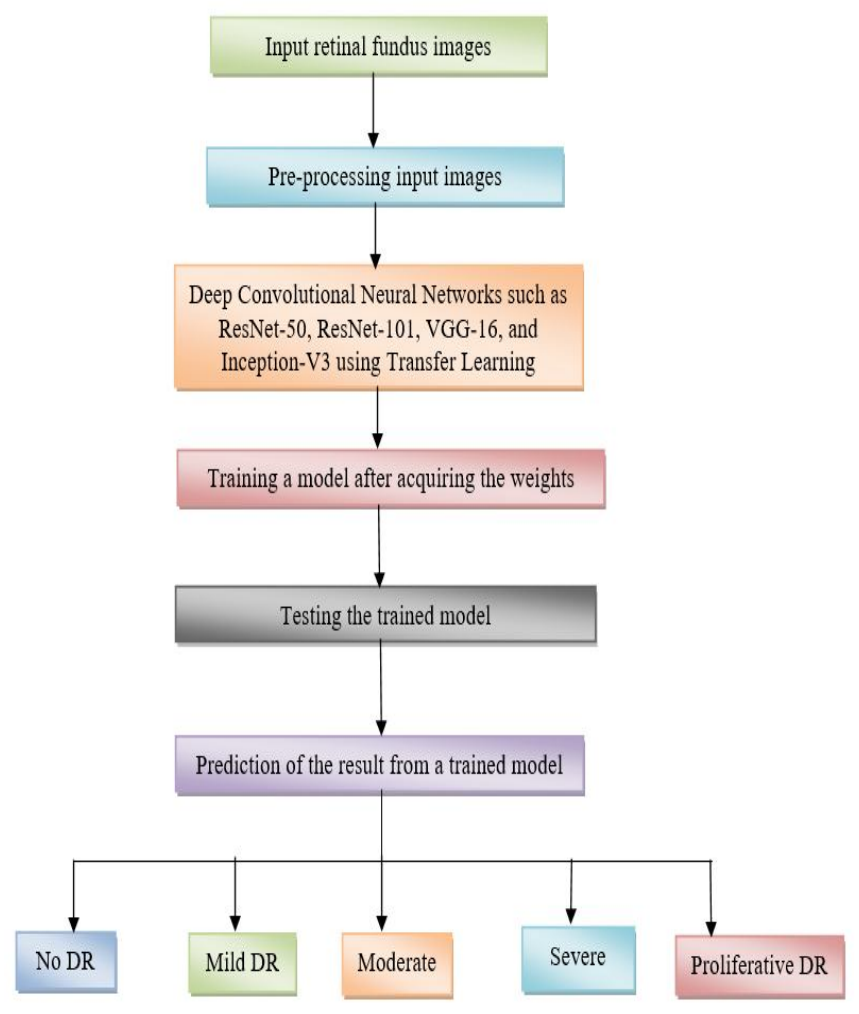

Figure 3: Flow Diagram for proposed methodology based on transfer learning fine-tuning.

In this study, pre-trained networks from ImageNet used as shown in Figure 4. The networks contains input layer, convolution layers and activation functions followed by pooling layers, batch normalization is used and fully connected layers followed the softmax classification layer 
finally for the result classification into five classes. The weights were transferred from the pre-trained network, so no need to train the network from scratch. Dataset used is Kaggel diabetic retinopathy fundus images database. To Fine-tuning the network, extra layers are added on top the existing pre-trained network. One global spatial average pooling layer followed by that fully connected and dropout layers and an output dense layer with softmax classifier is added to classify images into four classes. Fully connected layer with ReLu or LeakyReLu activation function then Dropout layer with 0.5 probability are added to the pre-trained neural network.

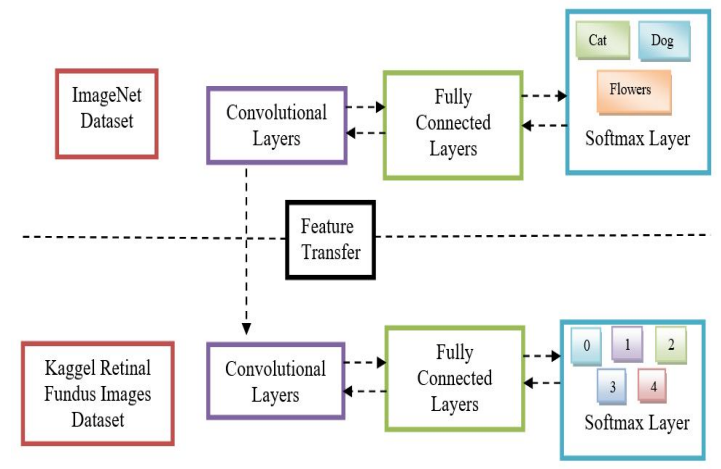

Figure 4: Deep Convolutional Neural Network Training using Transfer Learning.

\section{RESULT AND ANALYSIS}

In this research, it is not only important to get good training and test accuracy but also to find the number of accurately and inaccurately classified test images. Not only important to measure the number of accurately and inaccurately classified test images, but also to estimate how many fundus images are misclassified.The images are mainly classified into five classes based on the symptoms rate class can estimated by the feature called Hemorrhages, Exudates, Microaneurysms and Optic disc.The dataset was splitted into $80 \%$ for training and $20 \%$ for testing for the purpose of cross valiadtion. Experimantaion was conducted by using different activation functions on the above mentioned Architectures. The dataset was taken as 40 batchsize and trained for 40 epocs for eachof the architectures. The results got from the experimental study are shown below. The Figure.4. shows the results obtained from ResNet-50 with ReLu activation function and after fine-tune gave best results. Figure.5. shows the results from ResNet-101 using ReLu as activation function, fine tuning achieved good results. In Figure.6. it is observed that VGG16 after finetuning with ReLu activation function achieved best result. Inception-V3 gave best results with ReLu activation function without finetuning shown in Figure 7. The results achieved in this experimentation are shown in the below Table 1 and Table 2. The tables contains the results achieved before finetuning and after finetuning with useof Relu and LeakyRelu activation function. Figure 9. Shows the confusion matrix got from the experimentation with different neural networks.

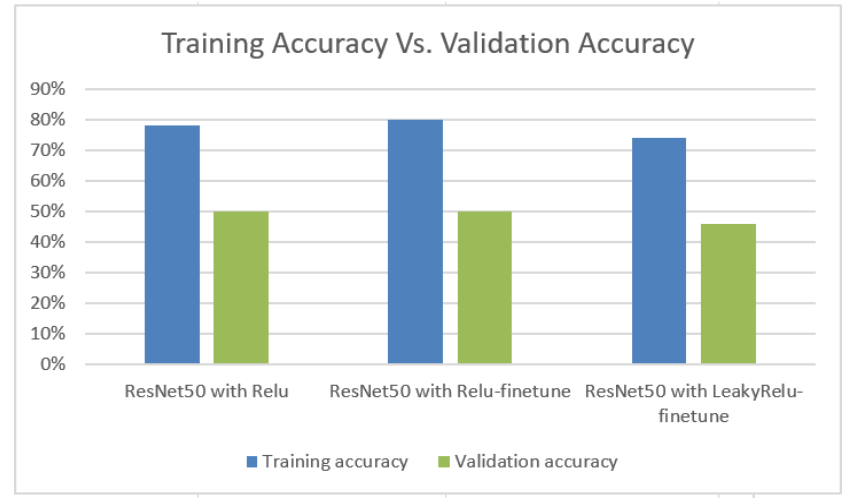

Figure 5: ResNet50 Results.

Training Accuracy Vs. Validation Accuracy

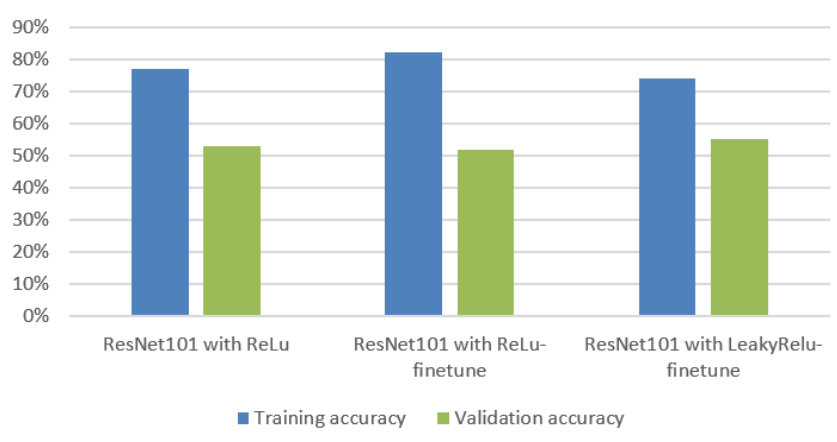

Figure 6: ResNet101 Results.

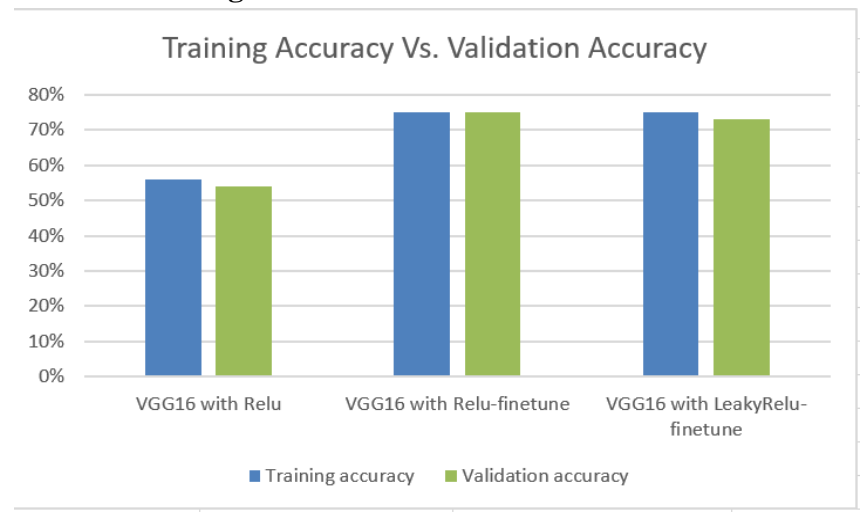

Figure 7: VGG16 Results.

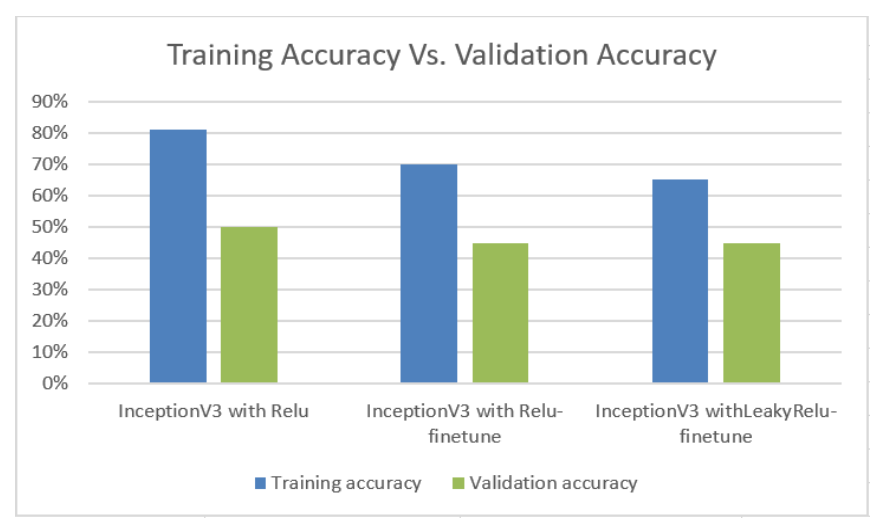

Figure 8: InceptionV3 Results 
Kusuma R C et al., International Journal of Advanced Trends in Computer Science and Engineering, 9(4), July - August 2020, 5009 - 5014

Table 1: Training and Vlidation Accuracies for CNN Different Architectures with fine-tuning.

\begin{tabular}{|c|c|c|}
\hline CNN Architectures & $\begin{array}{l}\text { Training } \\
\text { Accuracy With } \\
\text { Fine Tuning }\end{array}$ & $\begin{array}{l}\text { Validation } \\
\text { Accuracy With Fine } \\
\text { Tuning }\end{array}$ \\
\hline $\begin{array}{c}\text { ResNet50 with ReLu } \\
\text { Activation Function }\end{array}$ & $80 \%$ & $50 \%$ \\
\hline $\begin{array}{c}\text { ResNet101 with ReLu } \\
\text { Activation Function }\end{array}$ & $82 \%$ & $52 \%$ \\
\hline $\begin{array}{c}\text { VGG16 with ReLu } \\
\text { Activation Function }\end{array}$ & $75 \%$ & $75 \%$ \\
\hline $\begin{array}{c}\text { InceptionV3 with ReLu } \\
\text { Activation Function }\end{array}$ & $81 \%$ & $45 \%$ \\
\hline $\begin{array}{c}\text { ResNet50 with } \\
\text { LeakyReLu Activation } \\
\text { Function }\end{array}$ & $74 \%$ & $46 \%$ \\
\hline $\begin{array}{c}\text { ResNet101 with } \\
\text { LeakyReLu Activation } \\
\text { Function }\end{array}$ & $74 \%$ & $55 \%$ \\
\hline $\begin{array}{c}\text { VGG16 with } \\
\text { LeakyReLu Activation } \\
\text { Function }\end{array}$ & $75 \%$ & $73 \%$ \\
\hline $\begin{array}{c}\text { InceptionV3 with } \\
\text { LeakyReLu Activation } \\
\text { Function }\end{array}$ & $65 \%$ & \\
\hline
\end{tabular}

Table 2: Training and Vlidation Accuracies for CNN Different Architectures without fine-tuning.

\begin{tabular}{|c|c|c|}
\hline $\begin{array}{c}\text { CNN } \\
\text { Architectures }\end{array}$ & $\begin{array}{c}\text { Training Accuracy } \\
\text { Without Fine } \\
\text { Tuning }\end{array}$ & $\begin{array}{c}\text { Validation } \\
\text { Accuracy Without } \\
\text { Fine Tuning }\end{array}$ \\
\hline $\begin{array}{c}\text { ResNet50 with ReLu } \\
\text { Activation Function }\end{array}$ & $78 \%$ & $50 \%$ \\
\hline $\begin{array}{c}\text { ResNet101 with ReLu } \\
\text { Activation Function }\end{array}$ & $77 \%$ & $53 \%$ \\
\hline $\begin{array}{c}\text { VGG16 with ReLu } \\
\text { Activation Function }\end{array}$ & $56 \%$ & $54 \%$ \\
\hline $\begin{array}{c}\text { InceptionV3 with } \\
\text { ReLu Activation } \\
\text { Function }\end{array}$ & $70 \%$ & $50 \%$ \\
\hline
\end{tabular}
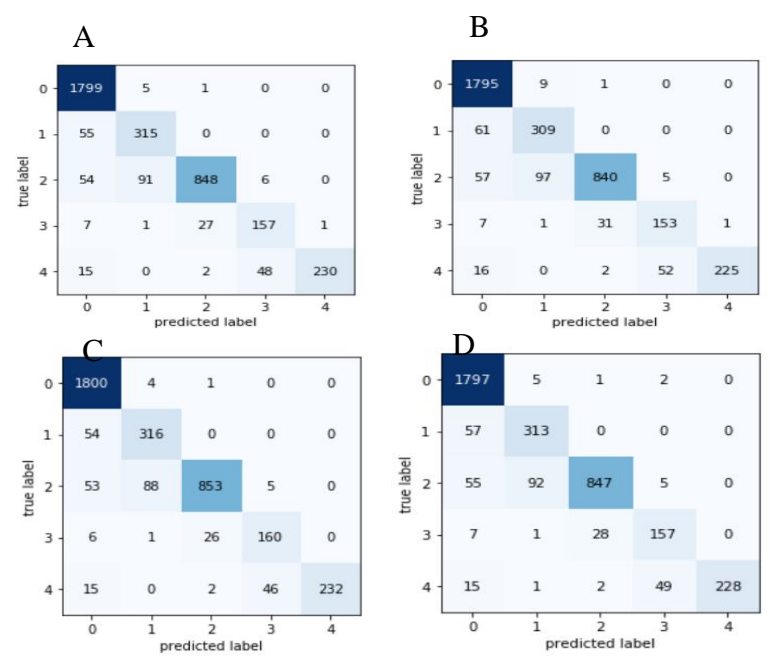

Figure 9: Confusion matrix (A)VGG16 (B)ResNet50 (C)ResNet101 (D)InceptionV3 on test Dataset

\section{CONCLUSION}

$\mathrm{DR}$ (Diabetic Retinopathy) is quite common disease these days in the peoples over the age of 60 . This one of the main reason for Blindness in Diabetic patients. It is very imporatnt to provide some fruitful technologies for diabetic retinopathy detection in early stages. Using the retinal fundus images the features are extracted automatically. The computer-adied technique will effectively aid the Clinicians for diagnosis. So by using this technologies the chances of Misdignosis will be very less whe compared to manual diagnosis.

In the proposed methodology DCNNs for detection of Diabetic Retinopathy using transfer learning are presented to classify Diabetic Retinopathy. Deeper the architecture will achieve better training result as compared to the shallow network. From the implementations and Result Analysis it can be concluded that After fine-tuning VGG16 is giving good training and validation accuracies. Without fine-tuning ResNet101 is achieved 0.82 percent of training and 0.52 percent of validation accuracy and VGG16 after finetuning giving 0.75 percentage of training and 0.75 percentage of validation accuracy.

\section{FUTURE WORK}

In the propose methodology pretrained networks are trained for the APTOS blind ness detection dataset. Transfer learning is used to avoid training the model from scratch and varied parameters to get good validation and training results. In future have to build own network for the Diabetic Retinoapthy prediction and varying of hyperparameters to get best accurate results both in terma of training and validation.

\section{ACKNOWLEDGEMENT}

The authors express their sincere gratitude to Prof. N R Shetty, Advisor and Dr. H C Nagaraj, Principal, Nitte Meenakshi Institute Of Technology for giving constant support to carry out research at NMIT.

\section{REFERENCES}

1. Feng Li, Zheng Liu, Hua Chen, Minshan Jiang, Xuedian Zhang, Zhizheng Wu. Automatic Detection of Diabetic Retinopathy in Retinal Fundus Photographs Based on Deep Learning Algorithm, Published online 2019 Nov 12. https://doi.org/10.1167/tvst.8.6.4

2. Zhentao Gao; Jie Li; Jixiang Guo; Yuanyuan Chen; Zhang Yi; Jie Zhong. Diagnosis of Diabetic Retinopathy Using Deep Neural Networks, 3 October 2018.

3. Alex Tamkin, Iain Usiri, Chala Fufa. Deep CNNs for Diabetic Retinopathy Detection, Tamkin Stanford University.

4. Sarfaraz Masood, Tarun Luthra, Identification of Diabetic Retinopathy in Eye Images Using Transfer Learning, 2017 International Conference on Computing, Communication and Automation (ICCCA). 
5. Nour Eldeen Mahmoud Khalifa, Mohamed Loey, Mohamed Hamed N. Taha, Mohamed Hamed N. Taha Deep Transfer Learning Models for Medical Diabetic Retinopathy Detection, Inform Med. 2019 Dec.

6. Et, al. Nikhil M N, Angel Rose A, Diabetic Retinopathy Stage Classification using CNN, International Research Journal of Engineering and Technology (IRJET) 03 May 2019.

7. D. Jude Hemanth, Omer Deperlioglu, Utku Kose, An enhanced diabetic retinopathy detection and classification approach using deep convolutional neural network, December 2019.

8. Misgina Tsighe Hagos, Shri Kant, Transfer Learning based Detection of Diabetic Retinopathy from Small Dataset, 22 May 2019.

9. Muhammad Mateen, Junhao Wen, Dr Nasrullah, Song Sun, Exudate Detection for Diabetic Retinopathy Using Pretrained Convolutional Neural Networks, 10 April 2020.

10. Ravi M. Kamble; Genevieve C. Y. Chan; Oscar Perdomo; Manesh Kokare ; Fabio A. González; Henning Müll, Automated Diabetic Macular Edema (DME) Analysis using Fine Tuning with Inception-Resnet-v2 on OCT Images, 2018 IEEE-EMBS Conference on Biomedical Engineering and Sciences (IECBES).

11. Slavomír Kajan; Jozef Goga; Kristián Lacko; Jarmila Pavlovičová, Detection of Diabetic Retinopathy Using Pretrained Deep Neural Networks, 2018 The Institute of Electrical and Electronics Engineers (IEEE).

12. Yogesh Kumaran, Chandrashekar M. Patil, A Brief Review of the Detection of Diabetic Retinopathy in Human Eyes Using Pre-Processing \& Segmentation Techniques, International Journal of Recent Technology and Engineering (IJRTE), December 2018.

13. M. Mateen, J. Wen, Nasrullah, S. Song, and Z. Huang, Fundus image classification using VGG-19 architecture with PCA and SVD, Symmetry, vol. 11, no. 1, 2019. https://doi.org/10.3390/sym11010001

14. Saaddine, J.B., Honeycutt, A.A., Narayan, K.V., Zhang, X., Klein, R. and Boyle, J.P. Projection of diabetic retinopathy and other major eye diseases among people with diabetes mellitus, 2008. United States, 20052050. Archives of ophthalmology, 126(12), pp.1740-1747.

https://doi.org/10.1001/archopht.126.12.1740

15. Kaggle: Diabetic rentinopathy detection challenge. http://www.kaggle.com/c/diabetic-retinopathy-detection /data. 2016.

16. https://www.sciencedirect.com/science/article/pii/S1877 050916311929.

17. https://ysjournal.com/study-of-convolutional-neural-net works-for-early-detection-of-diabetic-retinopathy/

18. https://www.sciencedirect.com/science/article/pii/S0020 025519305377?casa_token=eigKiN_VrMMAAAAA:9t
5AzaX53QwV8zm0cbRWn2e8ezit15wg9mcb02yQU6qdTNTzeWSqdXSK7aHeJ9udI7vGF2vdxvu.

19. http://ebooks.iospress.nl/volumearticle/45311.

20. https://www.tensorflow.org/tutorials/images/transfer_le arning. 